\title{
Ado-Trastuzumab Emtansine-Induced Pulmonary Toxicity: A Single-Institution Retrospective Review
}

\author{
Heidi Egloff ${ }^{a}$ Kelley M. Kidwell ${ }^{b} \quad$ Anne Schott $^{a}$ \\ aUniversity of Michigan Rogel Cancer Center, Ann Arbor, MI, USA; ${ }^{b}$ Department of \\ Biostatistics, University of Michigan School of Public Health, Ann Arbor, MI, USA
}

\section{Keywords}

Ado-trastuzumab emtansine $\cdot$ T-DM1 $\cdot$ Pneumonitis $\cdot$ Pulmonary toxicity $\cdot$ Breast cancer

\begin{abstract}
Purpose: T-DM1 is an antibody drug conjugate with proven efficacy in metastatic breast cancer for progressive disease refractory to trastuzumab. Drug-induced pneumonitis is a rare serious potential adverse effect. The purpose of this review was to estimate the incidence of pulmonary toxicity at our institution. Methods: A retrospective analysis of electronic medical record data inclusive of all women and men aged 18 years and older treated with T-DM1 at out institution was undertaken. The records were reviewed for clinical symptoms and/or radiographic evidence concerning for pneumonitis. We identified variables of interest with regard to potential risk factors for toxicity. Results: A total of 50 patients were included, 6 (12\%) of whom had radiographic and/or clinical symptoms concerning for T-DM1-induced pneumonitis. All 6 patients had metastatic or unresectable breast cancer. Of the 6 patients, 5 (83\%) had suspected pulmonary metastases, $1(17 \%)$ had a history of underlying lung disease, and $5(83 \%)$ had a history of prior taxane therapy. Pulmonary metastases $(p=0.38)$, the median number of treatment cycles $(p=0.29)$, prior taxane therapy $(p=0.99)$, underlying lung disease $(p=0.99)$, and hormone receptor positivity $(p=0.66)$ did not have any statistical significance for an
\end{abstract}




\section{Case Reports in Oncology}

Case Rep Oncol 2018;11:527-533

DOI: $10.1159 / 000491574$

(c) 2018 The Author(s). Published by S. Karger AG, Base www.karger.com/cro

Egloff et al.: Ado-Trastuzumab Emtansine-Induced Pulmonary Toxicity:

A Single-Institution Retrospective Review

association with pneumonitis. Conclusion: Pneumonitis is a recognized toxic effect of T-DM1. While our sample size was small, the number of events was higher than described in the literature, which may be an artifact of referral bias. Future studies with a larger sample population may detect potential risk factors for toxicity.

(C) 2018 The Author(s)

Published by S. Karger AG, Basel

\section{Introduction}

Ado-trastuzumab emtansine (T-DM1) was the first antibody drug conjugate (ADC) to secure FDA approval in HER2-positive breast cancer. It consists of a monoclonal antibody, trastuzumab, bound by a thioether linkage to emtansine [1]. Emtansine (DM1) is a maytansinoid derivative, originally isolated in 1971 from the East African shrub Maytenus serrata $[2,3]$. Its mechanism of action is that of an inhibitor of the microtubule apparatus, similar to the vinca alkaloids, leading to eventual cell cycle arrest and significant cytotoxicity [4]. The ADC represents an efficient drug delivery system, whereby trastuzumab binds to the extracellular domain of HER2 expressed on breast cancer cells and subsequently signals uptake via endocytosis of the trastuzumab-emtansine complex. This targeted drug delivery is designed to minimize peripheral toxicity $[1,5]$. The thioether linkage and antibody component undergo lysosomal degradation when the endosome vesicle and lysosome fuse, allowing release of the DM1 component into the cell [6]. Meanwhile, the trastuzumab component continues to exert antitumor effects by way of cell signaling inhibition and antibody-directed cellular cytotoxicity [7].

T-DM1 has shown success in several clinical trials. Importantly, T-DM1 has proven efficacy in the setting of trastuzumab resistance. In comparison to the combination of lapatinib and capecitabine for advanced HER2-positive breast cancer in those who had progressed on trastuzumab and a taxane, T-DM1 demonstrated improved overall survival (30.9 vs. 25.1 months) with a higher objective response rate (43.6 vs. 30.8\%) in the EMILIA trial [8]. In the phase III TH3RESA trial, patients with advanced breast cancer who had progression on two or more HER2-directed therapies were randomized to receive T-DM1 versus chemotherapy of the physician's choice. At the second interim analysis for overall survival, T-DM1 demonstrated improved overall survival (22.7 vs. 15.8 months) in comparison [9].

The most common phase III study toxicities associated with T-DM1 are diarrhea, constipation, nausea, vomiting, fatigue, headache, epistaxis, peripheral neuropathy, arthralgia, pyrexia, thrombocytopenia, anemia, and elevated AST/ALT levels [8-10]. The current FDA prescribing information recommends discontinuation of T-DM1 in cases of interstitial lung disease or pneumonitis, and cites a pneumonitis incidence of $0.8 \%$ ( $7 / 884$ cases), with 1 case of grade 3 pneumonitis noted.

Anecdotal experience at our institution suggested the rate of pulmonary toxicity may be higher than reported in the clinical trials. We sought to determine the incidence of pulmonary toxicity, with a specific interest in the incidence of interstitial pneumonitis. Furthermore, variables of interest with regard to potential risk factors for pneumonitis were investigated. 


\section{Case Reports in Oncology}

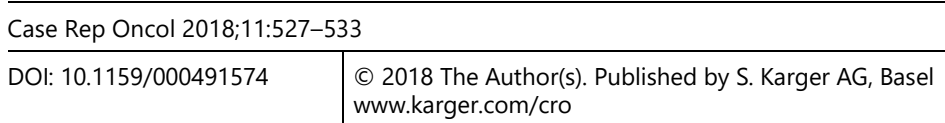

Egloff et al:: Ado-Trastuzumab Emtansine-Induced Pulmonary Toxicity:

A Single-Institution Retrospective Review

Methods

This study was designed as a retrospective chart review.

Patient Population

The subject population included all women and men aged 18 years or older who received at least one dose of T-DM1 at the University of Michigan Rogel Cancer Center facilities between January 1, 2013, and November 1, 2017. Patients with early-stage breast cancer and metastatic or locally advanced HER2-positive breast cancer were included.

\section{Data Acquisition}

Patients were identified upon review of pharmacy records for ado-trastuzumab emtansine drug administration. Electronic medical record numbers were matched to pharmacy records. The chart review of the electronic medical record data was performed by a single member of the study team. We defined the case incidence of interstitial pneumonitis among those patients who had a clinical picture (hypoxia, unexplained dyspnea, and exclusion of infection) and/or concerning imaging findings consistent with pneumonitis. Several variables of interest with regard to risk factors were abstracted from the chart, including the number of treatment cycles, suspicion of lung metastases, a history of lung disease, documented prior reaction to trastuzumab, and documented prior treatment with a taxane.

\section{Statistical Methods}

Due to the small number of pneumonitis events, the associations between the presence of pneumonitis and categorical clinical variables were assessed using Fisher's exact tests, and the associations between the presence of pneumonitis and continuous variables were assessed using the Wilcoxon rank-sum test.

\section{Results}

\section{Baseline Characteristics of the Patients}

Upon review of the pharmacy administration records, 50 unique patients were treated with at least one dose of T-DM1 at the Rogel Cancer Center from January 1, 2013, to November 1,2017 , all of whom were included in our analysis. The descriptive attributes of the study population are outlined in Table 1.

The majority of the patients $(43 / 50)$ had discontinued T-DM1 at the completion of the chart analysis. Treatment discontinuation was most commonly a result of progression $(n=29$; $65.9 \%)$; however, other reasons included any toxicity $(n=11 ; 27.2 \%)$, death as a result of progression $(n=2 ; 4.5 \%)$, and clinical trial treatment completion for early-stage breast cancer $(n=1 ; 2 \%)$.

\section{Treatment-Related Events and Risk Factor Analysis}

Of the analyzed population, 6 (12\%) had radiographic and/or clinical symptoms concerning for T-DM1-induced pneumonitis. All 6 patients identified with toxicity had metastatic or 
unresectable breast cancer. CTCAE v4.03 grade 3 pneumonitis (limiting activities of daily living, severe symptoms, and new oxygen requirement) was observed in $3 / 6$ patients.

On further analysis of those with clinical and/or radiographic symptoms of pneumonitis, $5(83 \%)$ had suspected pulmonary metastases, $1(17 \%)$ had a history of underlying lung disease, and $5(83 \%)$ had a history of prior taxane therapy.

Table 2 outlines the results for potential associations of the clinical variables with pneumonitis. Pulmonary metastases ( $p=0.38)$, the median number of treatment cycles $(p=0.29)$, prior taxane therapy ( $p=0.99)$, underlying lung disease $(p=0.99)$, and hormone receptor positivity $(p=0.66)$ did not have statistical significance for an association with pneumonitis. Time (in days) on T-DM1 did not predict pneumonitis ( $p=0.32$ ).

\section{Clinical Outcomes}

Reassuringly, all patients had significant resolution of their pneumonitis symptoms. Steroids were given in $4 / 6$ of the cases, with a time to complete resolution of 29.6 days (range 381).

\section{Discussion}

The goal of this review was to investigate the incidence of pneumonitis among breast cancer patients treated with T-DM1, while also examining potential risk factors and outcomes to guide toxicity identification and treatment in the future. Based on a review of the current published literature, this is the first review of interstitial pneumonitis in patients treated with T-DM1.

At least one prior study has investigated pneumonitis associated with trastuzumab monotherapy, with the conclusion that this is a rare adverse event $(0.5 \%)$ based on review of two large clinical trials (NSABP B-31 and N9831) [11]. Another case series described 3 patients who received adjuvant trastuzumab monotherapy and subsequently developed interstitial lung disease, which reassuringly resolved with drug discontinuation and steroids [12].

The currently available ADCs approved by the FDA include ado-trastuzumab emtansine, brentuximab vedotin, gemtuzumab ozogamicin, and inotuzumab ozogamicin, although only T-DM1 is approved for solid tumor malignancies. According to the FDA prescribing information, brentuximab vedotin was associated with a higher rate of noninfectious pulmonary toxicity; however, this was in combination with bleomycin, an agent with known pulmonary toxicity. A review of toxicities with regard to gemtuzumab ozogamicin and inotuzumab ozogamicin did not suggest increased pulmonary toxicity $[13,14]$. Lorvotuzumab mertansine (DM1) has been used as the cytotoxic agent in ADCs in prior clinical trials. A recent phase I/II study of DM1 in combination with carboplatin/etoposide in extensive-stage small cell lung cancer did not report pneumonitis as an adverse event [15]. Similarly, pneumonitis was not reported in phase I studies of cantuzumab mertansine in advanced solid tumors and of bivatuzumab mertansine in metastatic breast cancer and squamous cell carcinoma of the head/neck and esophagus [16-18]. Hence, the etiologic component and biologic mechanism responsible for inciting pneumonitis in patients receiving T-DM1 remains to be determined.

The diagnosis of interstitial pneumonitis is a challenging one, as competing diagnoses often delay initial recognition. The CTCAE v4.03 defines pneumonitis as "a disorder character- 
ized by inflammation focally or diffusely affecting the lung parenchyma." In this study, we used clinical symptoms including dyspnea, cough, fever, and hypoxia plus/minus imaging to identify index cases. This was further substantiated by excluding infectious causes and progressive metastatic disease. Reassuringly, all patients in this study had significant resolution of their symptoms with steroid initiation and cessation of therapy, except for 1 patient who continued on T-DM1 at the cutoff date of the analysis.

We explored several potential risk factors for pulmonary toxicity. Taxanes are recognized as a drug class associated with pulmonary toxicity; however, prior taxane use was not associated with a higher incidence of toxicity in this review. Prior reaction to trastuzumab appeared to be statistically significant in those who experienced pneumonitis, but this was a very small number of patients and limited based on review of the record for documentation of a prior reaction. It is difficult to draw a firm conclusion due to the limited sample size; however, it may suggest an immunoreaction to the antibody component as a potential etiology for development of symptoms. We hypothesized that the site of delivery of the ADC cytotoxic payload may influence toxicity, but those with lung metastases did not appear to have a statistically significantly higher rate of pneumonitis.

Limitations of our study include the small sample of patients from a single institution. Based on the pattern of referral to tertiary care centers, the incidence of pulmonary toxicity in this study may be overestimated in comparison to the general population. Future studies with larger sample sizes should focus on further investigation of the potential risk factors for this serious toxicity, as this would in turn allow for better patient selection and outcomes.

\section{Statement of Ethics}

The University of Michigan approved the study under IRB exemption, given the minimal risk to the subjects.

\section{Disclosure Statement}

We have no conflicts of interest to disclose.

\section{References}

1 Martínez MT, Pérez-Fidalgo JA, Martín-Martorell P, Cejalvo JM, Pons V, Bermejo B, et al. Treatment of HER2 positive advanced breast cancer with T-DM1: A review of the literature. Crit Rev Oncol Hematol. 2016 Jan;97:96-106.

2 Issell BF, Crooke ST. Maytansine. Cancer Treat Rev. 1978 Dec;5(4):199-207.

3 Lewis Phillips GD, Li G, Dugger DL, Crocker LM, Parsons KL, Mai E, et al. Targeting HER2-positive breast cancer with trastuzumab-DM1, an antibody-cytotoxic drug conjugate. Cancer Res. 2008 Nov;68(22): 9280-90.

4 Remillard S, Rebhun LI, Howie GA, Kupchan SM. Antimitotic activity of the potent tumor inhibitor maytansine. Science. 1975 Sep;189(4207):1002-5.

5 Krop I, Winer EP. Trastuzumab emtansine: a novel antibody-drug conjugate for HER2-positive breast cancer. Clin Cancer Res. 2014 Jan;20(1):15-20. 
6 Erickson HK, Park PU, Widdison WC, Kovtun YV, Garrett LM, Hoffman K, et al. Antibody-maytansinoid conjugates are activated in targeted cancer cells by lysosomal degradation and linker-dependent intracellular processing. Cancer Res. 2006 Apr;66(8):4426-33.

7 Junttila TT, Li G, Parsons K, Phillips GL, Sliwkowski MX. Trastuzumab-DM1 (T-DM1) retains all the mechanisms of action of trastuzumab and efficiently inhibits growth of lapatinib insensitive breast cancer. Breast Cancer Res Treat. 2011 Jul;128(2):347-56.

8 Verma S, Miles D, Gianni L, Krop IE, Welslau M, Baselga J, et al.; EMILIA Study Group. Trastuzumab emtansine for HER2-positive advanced breast cancer. N Engl J Med. 2012 Nov;367(19):1783-91.

9 Krop IE, Kim SB, Martin AG, LoRusso PM, Ferrero JM, Badovinac-Crnjevic T, et al. Trastuzumab emtansine versus treatment of physician's choice in patients with previously treated HER2-positive metastatic breast cancer (TH3RESA): final overall survival results from a randomised open-label phase 3 trial. Lancet Oncol. 2017 Jun;18(6):743-54.

10 Perez EA, Barrios C, Eiermann W, Toi M, Im YH, Conte P, et al. Trastuzumab Emtansine With or Without Pertuzumab Versus Trastuzumab Plus Taxane for Human Epidermal Growth Factor Receptor 2-Positive, Advanced Breast Cancer: Primary Results From the Phase III MARIANNE Study. J Clin Oncol. 2017 Jan;35(2):141-8.

11 Costa R, Costa-Filho RB, Talamantes SM, Queiroga F Jr, Campello EC, Cartaxo H, et al. Interstitial Pneumonitis Secondary to Trastuzumab: A Case Report and Literature Review. Case Rep Oncol. 2017 Jun;10(2):524-30.

12 Sugaya A, Ishiguro S, Mitsuhashi S, Abe M, Hashimoto I, Kaburagi T, et al. Interstitial lung disease associated with trastuzumab monotherapy: A report of 3 cases. Mol Clin Oncol. 2017 Feb;6(2):229-32.

13 Larson RA, Sievers EL, Stadtmauer EA, Löwenberg B, Estey EH, Dombret H, et al. Final report of the efficacy and safety of gemtuzumab ozogamicin (Mylotarg) in patients with CD33-positive acute myeloid leukemia in first recurrence. Cancer. 2005 Oct;104(7):1442-52.

14 Kantarjian HM, DeAngelo DJ, Stelljes M, Martinelli G, Liedtke M, Stock W, et al. Inotuzumab Ozogamicin versus Standard Therapy for Acute Lymphoblastic Leukemia. N Engl J Med. 2016 Aug;375(8):740-53.

15 Socinski MA, Kaye FJ, Spigel DR, Kudrik FJ, Ponce S, Ellis PM, et al. Phase 1/2 Study of the CD56-Targeting Antibody-Drug Conjugate Lorvotuzumab Mertansine (IMGN901) in Combination With Carboplatin/Etoposide in Small-Cell Lung Cancer Patients With Extensive-Stage Disease. Clin Lung Cancer. 2017 Jan;18(1):68-76.e2.

16 Helft PR, Schilsky RL, Hoke FJ, Williams D, Kindler HL, Sprague E, et al. A phase I study of cantuzumab mertansine administered as a single intravenous infusion once weekly in patients with advanced solid tumors. Clin Cancer Res. 2004 Jul;10(13):4363-8.

17 Rupp U, Schoendorf-Holland E, Eichbaum M, Schuetz F, Lauschner I, Schmidt P, et al. Safety and pharmacokinetics of bivatuzumab mertansine in patients with CD44v6-positive metastatic breast cancer: final results of a phase I study. Anticancer Drugs. 2007 Apr;18(4):477-85.

18 Tijink BM, Buter J, de Bree R, Giaccone G, Lang MS, Staab A, et al. A phase I dose escalation study with anti-CD44v6 bivatuzumab mertansine in patients with incurable squamous cell carcinoma of the head and neck or esophagus. Clin Cancer Res. 2006 Oct;12(20 Pt 1):6064-72.

Table 1. Baseline characteristics of the subjects $(n=50)$

$\begin{array}{ll}\text { Mean age, years } & 57 \\ \text { Hormone receptor positive } & 31(62 \%) \\ \text { HER2 positive } & 50(100 \%) \\ \text { Median number of treatment cycles } & 8.5 \\ \quad \text { Range } & 1-33 \\ \text { Suspected lung metastases } & 29(58 \%) \\ \text { Underlying lung disease } & 9(18 \%) \\ \text { Documented history of reaction to trastuzumab } & 2(4 \%)\end{array}$




\section{Case Reports in Oncology}

Case Rep Oncol 2018;11:527-533

DOI: $10.1159 / 000491574$

(C)

(C) 2018 The Author(s). Published by S. Karger AG, Basel www.karger.com/cro

Egloff et al.: Ado-Trastuzumab Emtansine-Induced Pulmonary Toxicity:

A Single-Institution Retrospective Review

Table 2. Association with pneumonitis

\begin{tabular}{lll}
\hline Variable & $\begin{array}{l}\text { Frequency of } \\
\text { pneumonitis, } n \text { (\%) }\end{array}$ & $p$ value \\
\hline $\begin{array}{l}\text { Hormone receptor positive } \\
\quad \text { No }\end{array}$ & $3(15.8)$ & 0.66 \\
$\quad$ Yes & $3(9.7)$ & \\
$\begin{array}{l}\text { Suspected lung metastases } \\
\text { No }\end{array}$ & $1(4.8)$ & 0.38 \\
$\quad$ Yes & $5(17.2)$ & \\
Underlying lung disease & & 0.99 \\
$\quad$ No & $5(12.2)$ & \\
$\quad$ Yes & $1(11.1)$ & 0.012 \\
Prior reaction to Herceptin & & \\
$\quad$ No & $4(8.3)$ & 0.99 \\
$\quad$ Yes & $2(100)$ & \\
Taxane & $1(9.1)$ & \\
$\quad$ No & $5(12.8)$ & \\
$\quad$ Yes &
\end{tabular}

